\title{
Estimating species sensitivity distributions on the basis of readily obtainable descriptors and toxicity data for three species of algae, crustaceans, and fish
}

\author{
Yuichi Iwasaki ${ }^{\text {Corresp., } 1}$, Kiyan Sorgog ${ }^{1}$ \\ ${ }^{1}$ Research Institute of Science for Safety and Sustainability, National Institute of Advanced Industrial Science and Technology, Tsukuba, Ibaraki, Japan \\ Corresponding Author: Yuichi Iwasaki \\ Email address: yuichiwsk@gmail.com
}

Estimation of species sensitivity distributions (SSDs) is a crucial approach to predicting ecological risks and water quality benchmarks, but the amount of data required to implement this approach is a serious constraint on the application of SSDs to chemicals for which there are few or no toxicity data. The development of statistical models to directly estimate the mean and standard deviation (SD) of the logarithms of log-normally distributed SSDs has recently been proposed to overcome this problem. To predict these two parameters, we developed multiple linear regression models that included, in addition to readily obtainable descriptors, the mean and SD of the logarithms of the concentrations that are acutely toxic to one algal, one crustacean, and one fish species, as predictors. We hypothesized that use of the three species' mean and SD would improve the accuracy of the predicted means and SDs of the logarithms of the SSDs. We derived SSDs for 60 chemicals based on quality-assured acute toxicity data. Forty-five of the chemicals were used for model fitting, and 15 for external validation. Our results supported previous findings that models developed on the basis of only descriptors such as log $K_{\text {ow }}$ had limited ability to predict the mean and SD of SSD (e.g., $r^{2}=0.62$ and 0.49 , respectively). Inclusion of the three species' mean and SD, in addition to the descriptors, in the models markedly improved the predictions of the means and SDs of SSDs (e.g., $r^{2}=0.96$ and 0.75, respectively). We conclude that use of the three species' mean and SD is promising for more accurately estimating an SSD and thus the hazardous concentration for $5 \%$ of species in cases where limited ecotoxicity data are available. 
1 Estimating species sensitivity distributions on the basis of readily obtainable descriptors and

2 toxicity data for three species of algae, crustaceans, and fish

3

4 Yuichi Iwasaki* and Kiyan Sorgog

5

6 Research Institute of Science for Safety and Sustainability, National Institute of Advanced

7 Industrial Science and Technology, Tsukuba, Ibaraki, Japan

8

9 Corresponding Author:

10 Yuichi Iwasaki

11 16-1 Onogawa, Tsukuba, Ibaraki 305-8569, Japan

12 Email address: yuichiwsk@gmail.com 


\section{Abstract}

14 Estimation of species sensitivity distributions (SSDs) is a crucial approach to predicting

15 ecological risks and water quality benchmarks, but the amount of data required to implement this

16 approach is a serious constraint on the application of SSDs to chemicals for which there are few

17 or no toxicity data. The development of statistical models to directly estimate the mean and

18 standard deviation (SD) of the logarithms of log-normally distributed SSDs has recently been

19 proposed to overcome this problem. To predict these two parameters, we developed multiple

20 linear regression models that included, in addition to readily obtainable descriptors, the mean and

21 SD of the logarithms of the concentrations that are acutely toxic to one algal, one crustacean, and

22 one fish species, as predictors. We hypothesized that use of the three species' mean and SD

23 would improve the accuracy of the predicted means and SDs of the logarithms of the SSDs. We

24 derived SSDs for 60 chemicals based on quality-assured acute toxicity data. Forty-five of the

25 chemicals were used for model fitting, and 15 for external validation. Our results supported

26 previous findings that models developed on the basis of only descriptors such as $\log K_{\mathrm{OW}}$ had

27 limited ability to predict the mean and SD of SSD (e.g., $r^{2}=0.62$ and 0.49 , respectively).

28 Inclusion of the three species' mean and SD, in addition to the descriptors, in the models

29 markedly improved the predictions of the means and SDs of SSDs (e.g., $r^{2}=0.96$ and 0.75 ,

30 respectively). We conclude that use of the three species' mean and SD is promising for more

31 accurately estimating an SSD and thus the hazardous concentration for $5 \%$ of species in cases

32 where limited ecotoxicity data are available.

\section{Keywords}

35 Species sensitivity distribution; Quantitative structure-activity relationship; Hazard/risk assessment; Predictive toxicity; Ecological risk assessment 


\section{INTRODUCTION}

38 In the ecological risk assessment of chemicals, the use of species sensitivity distributions (SSDs)

39 is fundamental to deriving "safe" concentrations such as the predicted no effect concentration

40 (PNEC) and water quality benchmarks, as well as the potentially affected percentages of species

41 (Vaal et al., 1997; Posthuma, Suter \& Traas, 2002; Iwasaki et al., 2015; Belanger et al., 2017;

42 Carr \& Belanger, 2019; Posthuma et al., 2019; Sorgog \& Kamo, 2019). SSDs can be derived on

43 the basis of acute or chronic toxicity data, or both. To derive an SSD for a given chemical, a set

44 of toxicity data is fitted to statistical distributions such as a log-normal or log-logistic

45 distribution. Therefore, toxicity results for multiple species are required, and the minimum

46 sample sizes (i.e., the minimum number of species required) typically vary between five and 10 ,

47 depending on the regulatory jurisdiction (Belanger et al., 2017). This data-demanding feature is a

48 critical barrier to the application of SSD to chemicals for which there are few, or no, toxicity data

49 (Hoondert et al., 2019; Hiki \& Iwasaki, 2020).

50 To address this data-demand issue, the development of statistical models with

51 physicochemical descriptors to estimate the mean and standard deviation (SD) of the logarithms

52 of log-normally distributed SSDs has recently been proposed (Hoondert et al., 2019).

53 Development of separate models for the mean and SD of the logarithms of SSDs rather than

54 models that estimate the hazardous concentration for $\mathrm{X} \%$ of species $\left(\mathrm{HC}_{\mathrm{x}}\right.$; typically, $\left.\mathrm{HC}_{5}\right)$ based

55 on an SSD is valuable for two different reasons. First, both of these parameters are required to

56 estimate the fraction of species affected by a given concentration of a toxic substance as well as

57 the concentration that is hazardous for a given percent of the species (Posthuma, Suter \& Traas,

58 2002). Second, the mean and SD have inherently different statistical properties (e.g., the SD of

59 the logarithms of the SSD is related to inter-species differences in sensitivity). Although the

60 results are preliminary, the coefficients of determination $\left(R^{2}\right)$ of models developed for the SDs of 
61 the logarithms of the SSDs were less than 0.4 (Hoondert et al., 2019). However, the parameters

62 that characterize SSDs can be more robust than the concentrations that are acutely toxic to

63 individual species (Iwasaki et al., 2015). A modeling approach aimed at characterizing SSDs

64 therefore seemed worth pursuing.

65 The major concept that we tested here was to make use of the mean and SD of toxicity

66 values for three biological species chosen (randomly) from three biological groups (algae,

67 crustaceans, and fish), in addition to readily obtainable descriptors, to improve the model

68 prediction of SSDs. This idea is similar to the application of QAAR (quantitative activity-

69 activity relationship) and QSAAR (quantitative structure-activity-activity relationship) models

70 (Furuhama, Hayashi \& Yamamoto, 2019). Acquiring at least one set of toxicity data from each

71 of algae, crustaceans, and fish may not be feasible for many chemicals, but these biological

72 groups are often the most representative in terms of data availability (Aurisano et al., 2019).

73 Given the difficulties in predicting SSD parameters on the basis of readily obtainable descriptors

74 alone (Hoondert et al., 2019), an approach that lies in between prediction based on "only

75 descriptor-based" models and prediction based only on experimentally obtained toxicity data

76 could be promising.

77 Here, acute toxicity data were used to estimate SSDs because of the paucity of quality-

78 assured acute toxicity data. Our specific aims were threefold (Figure 1). We first aimed to

79 develop and test linear regression models with readily obtainable descriptors, such as the $\log _{10^{-}}$

80 transformed octanol-water partition coefficient $\left(\log K_{\mathrm{OW}}\right)$, to predict the means and SDs of acute

81 log-normal SSDs. By doing this, we evaluated the previous conclusions of Hoondert et al.

82 (2019) about model performance. Second, we aimed to develop linear regression models with

83 predictors of means and SDs estimated on the basis of toxicity values for three species chosen

84 from three biological groups (algae, crustaceans, and fish). Finally, we aimed to develop and test 
85 linear regression models with predictors of means and SDs based on the three species' toxicity

86 data, as well as the descriptors, to predict the means and SDs of SSDs. Although several studies

87 have derived SSDs based on three species' toxicity data and have assessed the associated

88 uncertainties of $\mathrm{HC}_{50}$ and $\mathrm{HC}_{5}$, particularly in the field of life-cycle impact assessment (van Zelm

89 et al., 2007; Golsteijn et al., 2012; Douziech et al., 2020), to our knowledge no attempt has been

90 made to develop and test the models by the combined use of the two different types of predictors

91 (i.e., descriptors and three species' mean and SD).

92

MATERIALS AND METHODS

94

95

96

97

98

\section{Data collection}

All acute toxicity data (e.g., the 50\% effect concentration $\left[\mathrm{EC}_{50}\right]$ and median lethal concentration $\left.\left[\mathrm{LC}_{50}\right]\right)$ were collected from datasets used for initial environmental risk assessments (IERAs) of chemicals; these have been performed annually from 2002 by the Ministry of Environment (MoE), Japan (MoE Japan, 2018). We obtained some of the datasets (2002-2014) from the National Institute of Advanced Industrial Science and Technology (AIST)-Multi-purpose Ecological Risk Assessment and Management Tool (AIST-MeRAM; Copyright No: H28PRO2007; Lin et al. 2020), and we obtained the more recent datasets from original pdf documents (MoE Japan, 2018). In IERAs, the quality of the toxicity data collected is examined by referring to international or domestic test guidelines or by evaluating the test conditions, test species, and physicochemical properties of the chemical being tested. The quality is assigned to one of four categories (Reliable without restriction, Reliable with restrictions, Not reliable, and Not assignable) in accord with OECD (2007) and Klimisch, Andreae \& Tillmann (1997). Here, we used the toxicity data assigned to the former two categories, which were also used to derive PNECs in the original IERAs, to minimize undesired variability in toxicity data and thereby to 
109 accurately estimate the means and SDs of SSDs. The acute toxicity measures analyzed were

110 dominated by $\mathrm{EC}_{50} \mathrm{~s}$ and $\mathrm{LC}_{50} \mathrm{~s}$, but they also included other similar measures (e.g., median

111 tolerance limit $\left(\mathrm{TL}_{\mathrm{m}}\right)$ and $50 \%$ inhibitory concentration $\left.\left(\mathrm{IC}_{50}\right)\right)$. To estimate the SSD of a

112 chemical, if multiple toxicity data were available for a single species, the calculated geometric

113 mean was used (Iwasaki et al., 2015; Hiki \& Iwasaki, 2020).

114 To estimate log-normally distributed SSDs, we also used the following criteria: (1)

115 toxicity data for $\geq 8$ species, including at least one species from each of the three biological

116 groups (algae, crustaceans, and fish) were available; (2) the assumption of normality of SSD was

117 not rejected by the Shapiro-Wilk test ( $\alpha=0.05$ with Holm's $P$-value adjustment; Hiki \& Iwasaki

118 (2020)); and (3) all the information about the predictors below was acquired, excluding that for

119 inorganic substances. Application of these criteria yielded a total of 60 chemicals to be analyzed

120 (see Table S1 in the Supplementary File for the list of chemicals). Use of a relatively large

121 numbers of species (10-55) has been advocated for accurate estimation of SSDs (Newman et al.,

122 2000; Carr \& Belanger, 2019; Hiki \& Iwasaki, 2020), but a relatively small numbers of species

$123(<10)$ has been used previously, and a minimum sample size of 5 has been suggested (TenBrook

124 et al., 2009). However, if 10 species had been used as the threshold in the present study, the

125 number of chemicals analyzed would have been reduced by almost a factor of two. We therefore

126 used a minimum sample size of eight species as a tradeoff between the number of chemicals

127 analyzed and the uncertainties in the estimated SSDs. We were able to estimate 29 SSDs with

128 data on 8 or 9 species, and we could estimate 31 SSDs with information for at least 10 species

129 (see Table S1 in the Supplementary File for more details).

130 The means and SDs of the logarithms of the log-normally distributed SSDs for 60

131 chemicals were calculated based on full datasets. The means and SDs were obtained by

132 calculating the means and sample SDs of the $\log _{10}$-transformed toxicity data. A sample SD was 
133 equated to the square root of the sum of the squared deviations from the mean divided by $\mathrm{n}-1$,

134 where $n$ was the number of species. In the present study, we have referred to the statistics that we

135 calculated as means and SDs, although they were actually the means and SDs of the $\log _{10^{-}}$

136 transformed values. Those means and SDs were used as objective variables in the later

137 regression analysis. The $\log _{10}$-transformed values of $\mathrm{HC}_{5}$ were derived by using equation (1):

$$
H C_{5}=\text { mean }-1.645 \times S D
$$

139 The $95 \%$ confidence intervals of the $\log _{10}$-transformed $\mathrm{HC}_{5} \mathrm{~s}$ based on SSDs derived from

140 measured toxicity values were also estimated according to Aldenberg, Jaworska \& Traas (2002)

141 (see Sorgog \& Kamo 2019 for the R code). As with SSD means and SDs, we hereafter referred to $142 \log _{10}$-transformed $\mathrm{HC}_{5} \mathrm{~s}$ as $\mathrm{HC}_{5} \mathrm{~s}$.

Predictors

146 Molecular weight (MW) obtained from the IERA reports, as well as log $K_{\mathrm{OW}}$ (estimated on the

147 basis of KOWWIN v1.69), $\log _{10}$-transformed vapor pressure at $25{ }^{\circ} \mathrm{C}$ (units: $\mathrm{mm} \mathrm{Hg}$;

148 MPBPWIN v1.43; hereafter, $\log$ VP), and the calculated rating value for the time required for

149 primary biodegradation (BIOWIN4; BIOWIN v4.10) — all of which were estimated by using the

150 EPI (Estimation Programs Interface) Suite v4.11 (U. S. EPA, 2017)—were used as predictors in

151 the regression analyses. These predictors were selected on the basis of the results of Hoondert et 152 al. (2019) and Iwasaki \& Hayashi (2020). Water solubility was not used, because it was

153 estimated from $\log K_{\mathrm{OW}}(U . S . E P A, 2017)$ and was highly correlated with $\log K_{\mathrm{OW}}(r=-0.96)$.

154 As predictors for the regression analyses, we also used two binary variables (Class 3 and Class 4)

155 concerning whether a given chemical was categorized as Class 3 (reactive chemicals) or Class 4 
156 (specifically acting chemicals) on the basis of the Verhaar mode-of-action classification

157 (Verhaar, van Leeuwen \& Hermens, 1992), as estimated by using the OECD QSAR Toolbox

158 (v4.4.1; https://qsartoolbox.org/). This is because accumulated evidence indicates that the means 159 and SDs of SSDs for Class 3 and 4 chemicals differ from those of other chemicals (Vaal et al.,

160 1997; Hendriks et al., 2013; Hiki \& Iwasaki, 2020). Because the influences of chemical classes

161 other than Class 3 and Class 4 were not evident in those studies, we chose not to include a

162 categorical variable representing the Verhaar mode-of-action classification to save the degree of

163 freedom. Of the 60 chemicals that we studied, 27\% were assigned to Class 1 (narcosis or

164 baseline toxicity), $8 \%$ to Class 2 (less inert compounds), $37 \%$ to Class $3,12 \%$ to Class 4 , and

$16517 \%$ to Class 5 (unclassified; see Table S1 for the raw data).

166 For each chemical, we also calculated the mean and SD of the toxicity data for an alga,

167 crustacean, and fish and hereafter refer to these 3-species-based predictors as the 3-species mean 168 and SD, respectively. For purposes of this calculation, we first produced 3000 bootstrapped

169 sample sets of 3-species means and SDs for each chemical by randomly choosing three species

170 that had been tested (i.e., choosing one species from each of the three biological groups by non-

171 parametric bootstrapping). The median value of the 3-species means and the corresponding SD

172 were then selected for each chemical, and these were used as predictors based on the 3-species

173 data in the later analysis. Note that the three species adopted here were also included to calculate

174 the means and SDs of SSDs, which were used as objective variables in the regression analysis.

175 The use of the 3-species mean and SD as predictors in the regression analysis is unconventional,

176 because the toxicity data used for calculating the predictors were also utilized to calculate

177 objective variables. However, it should be plausible given the limited availability of toxicity data

178 and our focus on examining the utility of the predictors in estimating SSDs.

179

Peer] reviewing PDF | (2020:11:55879:2:0:NEW 20 Jan 2021) 
181 All statistical analyses were performed by using R 3.6.3 (R Core Team, 2020). All the associated

182 data and the R code are available in the Supplementary File. For model development and

183 evaluation, 45 chemicals were randomly chosen from the 60 chemicals and used as training data,

184 and the remaining data (i.e., 15 chemicals) were used as test data for out-of-bag model

185 evaluation. The median values and ranges of SSD means and SDs and the descriptors of the test

186 data were generally within those of the training data, although the minimum value of $\log K_{\mathrm{OW}}$ in

187 the test data was slightly lower (Table 1).

To predict the means and SDs of the SSDs, we developed three types of the two linear regression models, namely: (1) the best models with descriptors only; (2) the models with the 3-

190 species-based predictors only; and (3) the best models with both descriptors and 3-species-based

191 predictors. For the development of models (2) and (3), we used the 3-species mean and SD to

192 predict the means and SDs of the SSDs, respectively. The 3-species mean (or SD) was therefore

193 not used to predict the SSD SDs (or means). Also, for model developments (1) and (3), models

194 were selected on the basis of the corrected Akaike information criterion (AICc; Burnham \&

195 Anderson, 2002 and Burnham, Anderson \& Huyvaert, 2011) with the training data, by using the

196 "dredge" function in the R package "MuMIn." The best models with the smallest AICc values

197 were selected among all possible models considered. Although our major focus was on selecting

198 the best models for parsimonious predictions, full-model-averaged standardized parameter

199 estimates were calculated by using the "model.avg" function of "MuMIn" to examine the relative

200 variable importance (Galipaud, Gillingham \& Dechaume-Moncharmont, 2017). We also

201 performed complementary analyses by fitting the best models with both descriptors and 3-

202 species-based predictors to evaluate the robustness of the model selection results based on the 203 median estimates. In the analyses, instead of the median values of 3-species means and SDs, we 
204 used five sets of 3-species means and SDs. The five sets were randomly chosen from the 3000

205 bootstrapped samples, and we tested the hypothesis that the results of model selection and fitting

206 were unchanged by use of the five sets.

207 By referring to OECD (2017) and Furuhama, Hayashi \& Yamamoto (2018), as internal

208 validation we calculated two commonly used statistics - the coefficient of determination $\left(r^{2}\right)$ and

209 the root mean square error (RMSE) - to evaluate the goodness of fit of individual models, and

210 we determined the leave-one-out cross-validated coefficient of determination $\left(q^{2}\right)$ to evaluate the

211 model robustness by using the R package "caret” (Kuhn, 2008). In addition, as external

212 validation, we determined the RMSE (RMSE ${ }_{\text {ext }}$ ) of the test data, the squared correlation

213 coefficient between the measured and predicted values $\left(r^{2}{ }_{\text {ext }}\right)$, and the explained variance $\left(Q^{2}{ }_{\text {ext }}\right)$

214 of the test data. We also used the developed models to predict the means and SDs of SSDs and

215 then the $\mathrm{HC}_{5} \mathrm{~s}$, which were compared with $\mathrm{HC}_{5} \mathrm{~s}$ estimated on the basis of toxicity data. To

216 evaluate the uncertainty of the $\mathrm{HC}_{5}$ s predicted from the developed models, the $95 \%$ confidence

217 intervals were calculated using non-parametric bootstrapping (i.e., the 95\% confidence intervals

218 were equated to the $75^{\text {th }}$ and $2925^{\text {th }}$ of 3000 sorted $\mathrm{HC}_{5}$ s based on the best models fitted to 3000

219 resampled datasets).

220

221 RESULTS

222 Predictors included in the best model developed with descriptors alone for SSD mean were Class

$2234, \log K_{\mathrm{OW}}$, and Class 3, all of which had higher (negative) full-model-averaged standardized

224 parameter estimates than the other variables $(<-0.19$; Table 2$)$. Similarly, the best model

225 developed with descriptors alone for SSD SD included only Class 4. However, along with Class

226 4, Log VP and MW had higher full-model-averaged standardized parameter estimates than the

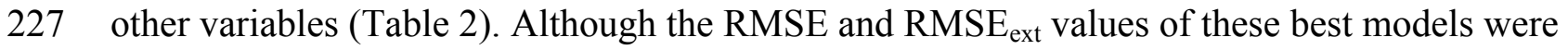


228 both below approximately 0.8 for the SSD mean and below approximately 0.2 for the SD (i.e.,

229 both within a factor of 10 of the values based only on measured toxicity data), the $r^{2}$ values were

230 low (below approximately 0.6 ; Table 3 ).

231 Incorporating the means or SDs of the toxicity values for three species markedly

232 improved the performances of the models (e.g., see changes in $r^{2}$ values in Table 3; Figure 2).

233 The best models for both descriptors and 3-species-based mean/SD were (see the Supplementary

234 File for more details):

235 SSD mean $=0.07+1.02 \times 3$ - species mean $-0.27 \times$ Class 4

236

$S S D S D=0.39+0.48 \times 3-$ species $\mathrm{SD}+0.30 \times$ Class $4+0.02 \times \log V P$

237 Our finding that the differences in AICc values between models with descriptors alone and those

238 with the 3-species mean or SD were over 20 also indicated the substantial model improvements

239 with the latter (Table 3; also see Burnham, Anderson \& Huyvaert, 2011 and Iwasaki \& Hayashi,

240 2020)). Particularly for the models for SSD mean, inclusion of only the 3-species mean as a

241 predictor increased the $r^{2}$ values from 0.62 to 0.96 , and this was also evident from the

242 prominently high full-model-averaged standardized parameter estimates of this predictor (i.e.,

243 0.94; Table 2). Inclusion of only the 3-species SD also improved the model fit in the prediction

244 of SSD SD (e.g., $r^{2}$ increased from 0.49 to 0.70 ; Table 3 ), and model performance was further

245 improved by including two descriptors (Log VP and Class 4; Tables 2 and 3). The best model for

246 predicting SSD mean had RMSE and $\mathrm{RMSE}_{\text {ext }}$ values of $<0.5$, and that for predicting SSD SD

247 had RMSE and RMSE ext $_{\text {values of }}<0.2$ (Table 3). Moreover, when we selected and evaluated

248 the best models by using five randomly chosen bootstrapped samples, the statistics for model

249 evaluation usually changed little (particularly, the coefficients of variation in the three statistics

250 for the internal validation $\left(r^{2}\right.$, RMSE, and $\left.q^{2}\right)$ were $<6 \%$ ), although the best models sometimes 
251 had one more, or one less, predictor compared with the best models developed above (see Tables

252 S2 and S3 in the Supplementary File).

253 As was expected from results discussed above, the $\mathrm{HC}_{5}$ values predicted from $\mathrm{SSD}$

254 means and SDs that were estimated on the basis of models with the 3-species mean/SD and the

255 best models developed with the 3-species mean/SD plus descriptors were highly correlated with

256 the $\mathrm{HC}_{5}$ values estimated on the basis of the measured toxicity data $\left(r^{2} \geq 0.95\right.$; Figure 3$)$, unlike

257 those based on the best models with descriptors alone $\left(r^{2}=0.67\right)$. Also, in the $\mathrm{HC}_{5}$ predictions

258 based on those models with the 3-species mean/SD, only one chemical (chloroform) was outside

259 a factor of 10 compared with the $\mathrm{HC}_{5}$ values derived from the measured data, whereas 15

260 chemicals were outside the range in the prediction based on models with descriptors alone

261 (Figure 3).

262

\section{DISCUSSION}

264 Our results based on SSDs derived for 60 chemicals clearly showed that the models developed

265 on the basis of descriptors alone had limited ability to predict the mean and SD of SSD and

266 thereby $\mathrm{HC}_{5}$; for example, this is particularly apparent from our finding that, when descriptors

267 alone were used, the predicted $\mathrm{HC}_{5}$ values for $25 \%$ of chemicals deviated by more than a factor

268 of 10 from $\mathrm{HC}_{5}$ values estimated on the basis of the measured toxicity data (Figure 3 ). These

269 results are generally consistent with the conclusion of Hoondert et al. (2019) that uncertainties

270 associated with prediction of the mean and $\mathrm{SD}$ of SSDs and $\mathrm{HC}_{5}$ by using descriptors may be

271 high (see Figure S3 of Hoondert et al. 2019 for the $\mathrm{HC}_{5}$ prediction). However, compared with

272 our results, the variations not explained by the models were somewhat larger in the work of

273 Hoondert et al. (2019). This difference was obvious from the $r^{2}$ values of their best models $(0.56$

274 and 0.29, respectively; see also Iwasaki \& Hayashi 2020), even though the predictors that they 
275 used were similar to ours, with the exception of the two binary variables (Class 3 and Class 4). It

276 is difficult to determine the underlying reasons for this difference in predictive ability. Two

277 apparent differences were the quantity of data and whether the quality of each of the

278 experimentally obtained toxicity values was examined. Hoondert et al. (2019) analyzed SSDs

279 based on acute toxicity data for 558 chemicals, which was approximately 9 times the number of

280 chemicals considered in the present study; however, they did not take the quality of each of the

281 experimentally obtained toxicity values into consideration although they differentiated the

282 quality of SSDs based on the inclusion of extrapolated toxicity data. Despite the clear trade-off,

283 both kinds of data should be valuable in developing predictive models that are well tested. There

284 is a need, however, for further testing of the utility of 3-species predictors with large datasets.

285 Compared with the models with descriptors alone, the addition of a 3-species mean/SD

286 markedly improved the predictions of the mean and SD of SSD (see Table 3), as well as of $\mathrm{HC}_{5}$.

287 These results suggest that using not only descriptors but also the mean and SD of toxicity data

288 for three species, each of which belongs to one of the three commonly tested biological groups

289 (algae, crustaceans, and fish), is a promising way of producing a more reliable SSD derivation in

290 cases where limited ecotoxicity data are available. Douziech et al. (2020) also demonstrated that

$291 \mathrm{HC}_{5}$ values estimated on the basis of acute toxicity data for three species of algae, daphnia, and

292 fish were mostly within a factor of 10 of $\mathrm{HC}_{5}$ values based on all measured toxicity values.

293 Although the uncertainties associated with our model estimates of $\mathrm{HC}_{5}$ were relatively small-

294 particularly for those obtained by including the 3-species mean/SD as a predictor (see the $\mathrm{x}$-axis

295 error bars in Figure 3), this prediction assumed that the means and SDs of SSDs calculated from

296 the measured toxicity data for at least 8 species were accurate. Caution is therefore required in

297 the use of such model-based estimates in ecological risk assessments because of the inherent

298 uncertainties in $\mathrm{HC}_{5}$ values based on measured toxicity data (y-axis error bars in Figure 3; see 
299 also de Zwart 2002 and Hiki \& Iwasaki 2020) as well as the large uncertainties in $\mathrm{HC}_{5}$ values

300 estimated on the basis of 3 species toxicity data (Douziech et al., 2020) .

301 The binary predictor, Class 4 (specifically acting chemicals), was included in all four

302 best models, with relatively large negative full-model-averaged standardized parameter estimates

303 in the models predicting SSD means and relatively large positive full-model-averaged

304 standardized parameter estimates in the models predicting SSD SDs (see Table 2). These results

305 support previous empirical evidence that specifically acting chemicals are generally more toxic

306 than other classes of chemicals, and that inter-species sensitivity to these chemicals varies more

307 widely than that to other classes of chemicals (Vaal et al., 1997; Hendriks et al., 2013; Hiki \&

308 Iwasaki, 2020); they also imply that such patterns cannot be fully captured by using only the 3-

309 species mean and SD. The variations in toxicity values within the biological groups appeared to

310 be relatively high for Class 4 chemicals (Figure S1), and this may have led to the importance of

311 Class 4 in the models that we developed. However, it is difficult to test this hypothesis robustly

312 owing to the limited number of Class 4 chemicals in our study (a total of 7 chemicals). Another

313 binary predictor, Class 3 (reactive chemicals), was selected in the best model with descriptors

314 alone for the SSD mean, and not in the other best models, suggesting that inclusion of the 3-

315 species mean could play an alternative role for Class 3 . The predictive abilities of the best

316 models developed with the 3-species mean/SD plus descriptors were similar among Classes 1-5

317 based on the Verhaar mode-of-action classification (see Figure S2 for the differences between

318 the predicted and measured means and SDs of the SSDs).

$319 \log K_{\mathrm{OW}}($ or $\log \mathrm{P})$ is commonly used in QSAR (quantitative structure-activity

320 relationship) models to predict ecotoxicity (Furuhama et al., 2010; Mayo-Bean et al., 2017), and

321 it was indeed included in the best model with descriptors alone for SSD mean. Log VP was

322 included in the best model with the 3-species SD plus descriptors (Table 2), and its regression 
323 coefficient was positive but non-significant $(P=0.072)$. This may indicate that the increased

324 variation in species sensitivity (i.e., the SD of SSD) was associated with the difficulty in toxicity

325 testing of the more volatile chemicals. However, a clear positive relationship between the SD of

326 SSD and log VP was not apparent owing to the large variation in the SD (Table S1). Note that

327 although use of estimated values of these descriptors is efficient, particularly if the number of

328 chemicals of concern is large, there are uncertainties associated with the estimated values (U.S.

329 EPA, 2007; Enoch et al., 2008) that may have affected the development of the model and the

330 accuracy of its predictions. Further tests of the developed models with experimentally obtained

331 values of descriptors would therefore be valuable, even though experimental results are

332 associated with uncertainties that can vary as a function of experimental conditions.

333

334 Conclusions

335 We demonstrated here that the use of measured toxicity data for three species selected from the

336 three commonly tested biological groups (algae, crustaceans, and fish) markedly improved the

337 model predictions for the means and SDs of log-normal SSDs based on acute toxicity.

338 Furthermore, the $\mathrm{HC}_{5} \mathrm{~s}$ in SSDs predicted by using such models were generally within a factor of

33910 of the values based only on measured toxicity data for at least 8 species. Therefore, the use of

3403 -species toxicity data could be promising for predicting the $\mathrm{HC}_{5}$ values from which PNECs are

341 frequently derived in ecological risk assessments. Note that our study was limited to log-normal

342 SSDs based on acute toxicity data for 60 chemicals, whereas PNECs for long-term exposure to

343 chemicals are derived from SSDs based on chronic toxicity data. However, because, for a large

344 proportion of chemicals, collecting chronic toxicity data for three species would be much more

345 difficult and less feasible than collecting acute toxicity data for three species (Hoondert et al.,

346 2019; Posthuma et al., 2019), other prediction approaches, including the use of acute to chronic

Peer] reviewing PDF | (2020:11:55879:2:0:NEW 20 Jan 2021) 
347 ratios for SSDs (de Zwart, 2002; Hiki \& Iwasaki, 2020) and in silico predictive models (Dyer et

348 al., 2008; Douziech et al., 2020; Takata et al., 2020), could be worth pursuing.

350 Acknowledgment

351 We are grateful to Takehiko Hayashi, Ayako Furuhama, Kyoshiro Hiki, Masashi Kamo, and

352 Wataru Naito for their useful comments and to Bin-Le Lin for her technical support. Comments

353 from Takashi Nagai originally inspired the present analysis.

354

355 References

356 Aldenberg T, Jaworska JS, and Traas TP. 2002. Normal species sensitivity distributions and

357 probabilistic ecological risk assessment. In: Posthuma L, Suter GW, and Traas TP, eds. Species

358 Sensitivity Distributions in Ecotoxicology. Boca Raton, FL, USA: Lewis Publishers, 49-102.

359 Aurisano N, Albizzati PF, Hauschild M, and Fantke P. 2019. Extrapolation factors for

360 characterizing freshwater ecotoxicity effects. Environmental Toxicology and Chemistry 38:2568-

$361 \quad 2582.10 .1002 /$ etc. 4564

362 Belanger S, Barron M, Craig P, Dyer S, Galay-Burgos M, Hamer M, Marshall S, Posthuma L,

363 Raimondo S, and Whitehouse P. 2017. Future needs and recommendations in the development of

364 species sensitivity distributions: Estimating toxicity thresholds for aquatic ecological communities

365 and assessing impacts of chemical exposures. Integrated Environmental Assessment and

366 Management 13:664-674. 10.1002/ieam.1841

367 Burnham KP, and Anderson DR. 2002. Model Selection and Multimodel Inference: A Practical

368 Information-Theoretic Approach. New York: Springer-Verlag. 
369 Burnham KP, Anderson DR, and Huyvaert KP. 2011. AIC model selection and multimodel

370 inference in behavioral ecology: some background, observations, and comparisons. Behavioral

371 Ecology and Sociobiology 65:23-35.

372 Carr GJ, and Belanger SE. 2019. SSDs revisited: Part I-A framework for sample size guidance

373 on species sensitivity distribution analysis. Environmental Toxicology and Chemistry 38:1514-

374 1525. 10.1002/etc. 4445

375 de Zwart D. 2002. Observed regularities in species sensitivity distributions for aquatic species. In:

376 Posthuma L, Traas TP, and Suter GW, eds. Species Sensitivity Distributions in Ecotoxicology.

377 Boca Raton, FL, USA: CRC Press, 133-154.

378 Douziech M, Ragas AMJ, van Zelm R, Oldenkamp R, Jan Hendriks A, King H, Oktivaningrum

379 R, and Huijbregts MAJ. 2020. Reliable and representative in silico predictions of freshwater 380 ecotoxicological hazardous concentrations. Environment International 134:105334.

381 https://doi.org/10.1016/j.envint.2019.105334

382 Dyer SD, Versteeg DJ, Belanger SE, Chaney JG, Raimondo S, and Barron MG. 2008. Comparison

383 of species sensitivity distributions derived from interspecies correlation models to sistributions

384 used to serive water quality criteria. Environmental Science \& Technology 42:3076-3083.

385 10.1021/es702302e

386 Enoch SJ, Hewitt M, Cronin MTD, Azam S, and Madden JC. 2008. Classification of chemicals

387 according to mechanism of aquatic toxicity: An evaluation of the implementation of the Verhaar

388 scheme in Toxtree. $\quad$ Chemosphere 243-248.

389 https://doi.org/10.1016/j.chemosphere.2008.06.052

390 Furuhama A, Hayashi TI, and Yamamoto H. 2018. Development of models to predict fish early-

391 life stage toxicity from acute Daphnia magna toxicity. SAR and QSAR in Environmental Research 392 29:725-742. 10.1080/1062936X.2018.1513423 
393 Furuhama A, Hayashi TI, and Yamamoto H. 2019. Development of QSAAR and QAAR models

394 for predicting fish early-life stage toxicity with a focus on industrial chemicals. SAR and QSAR in 395 Environmental Research 30:825-846. 10.1080/1062936X.2019.1669707

396 Furuhama A, Toida T, Nishikawa N, Aoki Y, Yoshioka Y, and Shiraishi H. 2010. Development

397 of an ecotoxicity QSAR model for the KAshinhou Tool for Ecotoxicity (KATE) system, March 3982009 version. SAR and QSAR in Environmental Research 21:403-413. $399 \quad 10.1080 / 1062936 X .2010 .501815$

400 Galipaud M, Gillingham MAF, and Dechaume-Moncharmont F-X. 2017. A farewell to the sum of 401 Akaike weights: The benefits of alternative metrics for variable importance estimations in model 402 selection. Methods in Ecology and Evolution 8:1668-1678. 10.1111/2041-210x.12835

403 Golsteijn L, Hendriks HWM, van Zelm R, Ragas AMJ, and Huijbregts MAJ. 2012. Do interspecies 404 correlation estimations increase the reliability of toxicity estimates for wildlife? Ecotoxicology and 405 Environmental Safety 80:238-243. https://doi.org/10.1016/j.ecoenv.2012.03.005

406 Hendriks AJ, Awkerman JA, de Zwart D, and Huijbregts MAJ. 2013. Sensitivity of species to 407 chemicals: Dose-response characteristics for various test types (LC50, LR50 and LD50) and 408 modes of action. Ecotoxicology and Environmental Safety 97:10-16. 409 http://dx.doi.org/10.1016/j.ecoenv.2013.06.020

410 Hiki K, and Iwasaki Y. 2020. Can we reasonably predict chronic species sensitivity distributions

411 from acute species sensitivity distributions? Environmental Science \& Technology 54:13131412 13136. 10.1021/acs.est.0c03108

413 Hoondert RPJ, Oldenkamp R, de Zwart D, van de Meent D, and Posthuma L. 2019. QSAR-based 414 estimation of species sensitivity distribution parameters: an exploratory investigation. 415 Environmental Toxicology and Chemistry 38:2764-2770. 10.1002/etc.4601 
416 Iwasaki Y, and Hayashi TI. 2020. Concerns about reproducibility, use of the Akaike information

417 criterion, and related issues in Hoondert et al. 2019. Environmental Toxicology and Chemistry

418 39:1300-1301. 10.1002/etc.4736

419 Iwasaki Y, Kotani K, Kashiwada S, and Masunaga S. 2015. Does the choice of NOEC or EC10

420 Affect the hazardous concentration for 5\% of the Species? Environmental Science \& Technology

421 49:9326-9330. 10.1021/acs.est.5b02069

422 Klimisch HJ, Andreae M, and Tillmann U. 1997. A Systematic Approach for Evaluating the

423 Quality of Experimental Toxicological and Ecotoxicological Data. Regulatory Toxicology and

424 Pharmacology 25:1-5. https://doi.org/10.1006/rtph.1996.1076

425 Kuhn M. 2008. Building predictive models in R using the caret package. Journal of Statistical

426 Software 28:1-26. 10.18637/jss.v028.i05

427 Lin B-L, Meng Y, Kamo M, and Naito W. 2020. An all-in-one tool for multipurpose ecological 428 risk assessment and management (MeRAM) of chemical substances in aquatic environment. 429 Chemosphere:128826. https://doi.org/10.1016/j.chemosphere.2020.128826

430 Mayo-Bean K, Moran-Bruce K, Meylan W, Ranslow P, Lock M, Nabholz V, Von Runnen J, 431 Cassidy L, and Tunkel J. 2017. Methodology document for the ecological structure-activity 432 relationship model (ECOSAR) class program, Ver 2.0. Office of Pollution Prevention and Toxics, 433 U.S. Environmental Protection Agency, Washington DC, USA.

434 MoE Japan. 2018. Profiles of the Initial Environmental Risk Assessment of Chemicals.

435 Newman MC, Ownby DR, Mézin LCA, Powell DC, Christensen TRL, Lerberg SB, and Anderson 436 B. 2000. Applying species-sensitivity distributions in ecological risk assessment: Assumptions of 437 distribution type and sufficient numbers of species. Environmental Toxicology and Chemistry 438 19:508-515. 10.1002/etc.5620190233 
439 OECD. 2007. Manual for Investigation for HPV Chemicals. Chapter 3: Data evaluation. Paris,

440 France. Available at http://www.oecd.org/chemicalsafety/risk-assessment/49191960.pdf

441 (accessed 2020-12-30)

442 OECD. 2017. Guidance on the Incorporation of Bioavailability Concepts for Assessing the

443 Chemical Ecological Risk and/or Environmental Threshold Values of Metals and Inorganic Metal

444 Compounds, OECD Series on Testing and Assessment, No. 259. Paris, France: OECD Publishing.

445 Posthuma L, Suter GWI, and Traas TP. 2002. Species Sensitivity Distributions in Ecotoxicology.

446 Boca Raton, FL: CRC Press.

447 Posthuma L, van Gils J, Zijp MC, van de Meent D, and de Zwart D. 2019. Species sensitivity 448 distributions for use in environmental protection, assessment, and management of aquatic 449 ecosystems for 12386 chemicals. Environmental Toxicology and Chemistry 38:905-917. $450 \quad 10.1002 /$ etc. 4373

451 R Core Team. 2020. R: A language and environment for statistical computing. Vienna, Austria. R 452 Foundation for Statistical Computing. Available at https://www.R-project.org/ (accessed 2020-12453 30)

454 Sorgog K, and Kamo M. 2019. Quantifying the precision of ecological risk: Conventional 455 assessment factor method vs. species sensitivity distribution method. Ecotoxicology and 456 Environmental Safety 183:109494. https://doi.org/10.1016/j.ecoenv.2019.109494

457 Takata M, Lin B-L, Xue M, Zushi Y, Terada A, and Hosomi M. 2020. Predicting the acute 458 ecotoxicity of chemical substances by machine learning using graph theory. Chemosphere 459 238:124604. https://doi.org/10.1016/j.chemosphere.2019.124604

460 TenBrook PL, Tjeerdema RS, Hann P, and Karkoski J. 2009. Methods for Deriving Pesticide 461 Aquatic Life Criteria. In: Whitacre DM, ed. Reviews of Environmental Contamination and 462 Toxicology Volume 199. Boston, MA: Springer US, 1-92. 
463 U. S. EPA. 2017. Estimation Programs Interface Suite ${ }^{\mathrm{TM}}$ for Microsoft ${ }^{\circledR}$ Windows, v 4.11. 464 Available at https://www.epa.gov/tsca-screening-tools/download-epi-suitetm-estimation465 program-interface-v411 (accessed 2020-12-30).

466 U.S. EPA. 2007. Science Advisory Board (SAB) Review of the Estimation Programs Interface 467 Suite (EPI Suite ${ }^{\mathrm{TM}}$ ). Science Advisory Board Science Advisory Board, U.S. Environmental 468 Protection Agency, Washington, DC, USA.

469 Vaal M, van der Wal JT, Hoekstra J, and Hermens J. 1997. Variation in the sensitivity of aquatic 470 species in relation to the classification of environmental pollutants. Chemosphere 35:1311-1327. 471 https://doi.org/10.1016/S0045-6535(97)00167-7

472 van Zelm R, Huijbregts MA, Harbers JV, Wintersen A, Struijs J, Posthuma L, and van de Meent 473 D. 2007. Uncertainty in msPAF-based ecotoxicological effect factors for freshwater ecosystems 474 in life cycle impact assessment. Integrated Environmental Assessment and Management 3:203475 210.10.1897/ieam_2006-013.1

476 Verhaar HJM, van Leeuwen CJ, and Hermens JLM. 1992. Classifying environmental pollutants. 477 Chemosphere 25:471-491. https://doi.org/10.1016/0045-6535(92)90280-5 
Figure 1

A summary schematic of model development in the present study.

Three types of predictive models were used to estimate means and standard deviations (SDs) of the logarithms of log-normally distributed species sensitivity distributions based on measured toxicity data.






\section{Figure 2}

Mean and standard deviation (SD) of the logarithms of log-normally distributed species sensitivity distributions (SSDs) estimated based on measured toxicity data alone or predicted from models developed in the present study.

$(A \& B)$ Mean and SD predicted from best models developed with descriptors alone. (C\&D) Mean and SD predicted from models developed with the 3-species mean/SD. (E \& F) Mean and SD predicted from best models developed with the 3-species mean/SD plus descriptors. See Tables 2 and 3 for details of best models and model evaluation, respectively. White and gray dots indicate training data and test data, respectively. 
Best models with only descriptors
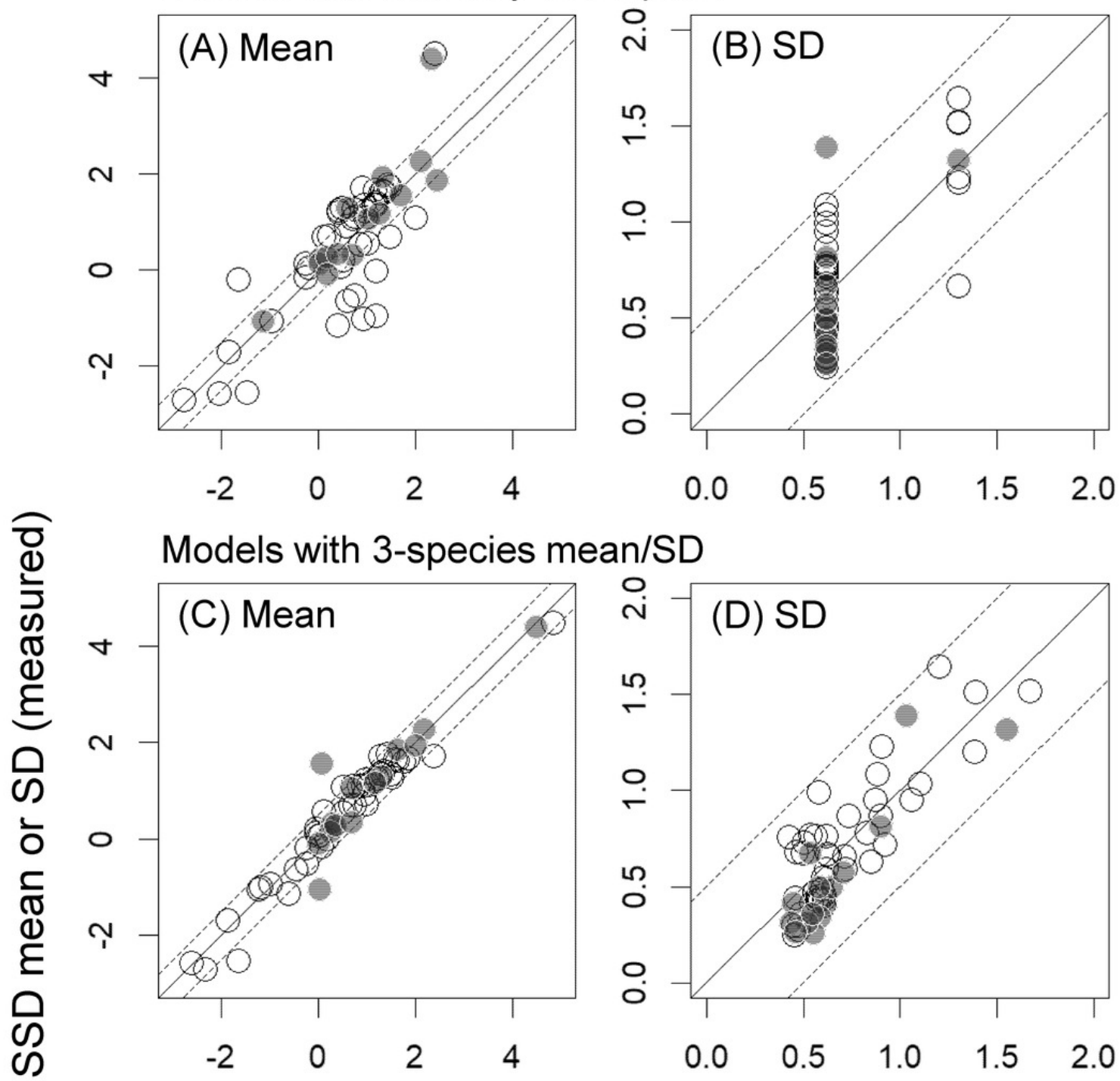

Best models with 3-species mean/SD plus descriptors






\section{Figure 3}

$\log _{10}$-transformed hazardous concentrations for $5 \%$ of species $\left(\mathrm{HC}_{5} \mathrm{~s}\right)$, estimated by species sensitivity distributions based on measured toxicity data or estimated by using three types of models ( $A-C$; see Table 2 for more information).

$\mathrm{X}$ - and $\mathrm{y}$-axis error bars respectively indicate $95 \%$ confidence intervals for $\mathrm{HC}_{5}$, as estimated by non-parametric bootstrapping, and those based on measured toxicity data (see the text for the details). White and gray dots indicate training data and test data, respectively. Values of $r^{2}$ indicate squared correlation coefficients between the measured and predicted $\mathrm{HC}_{5}$ values. SD = standard deviation.
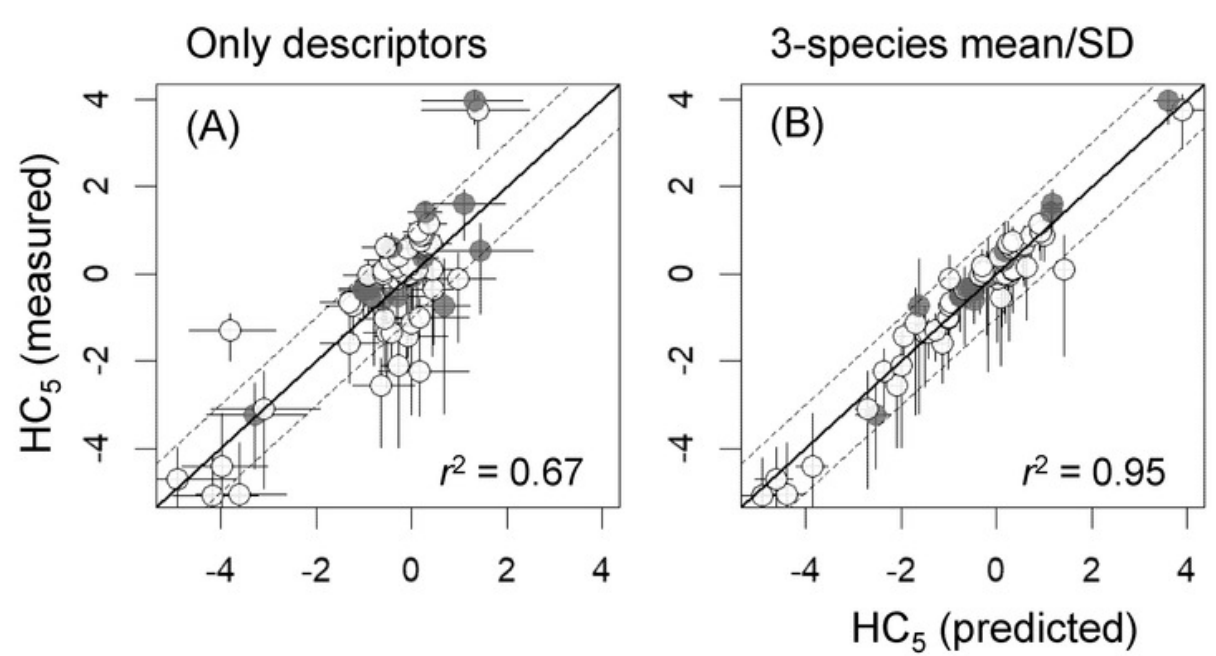

3-species mean/SD plus descriptors

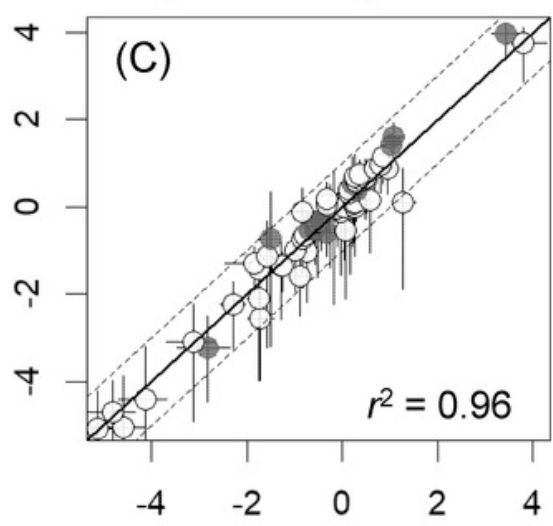




\section{Table $\mathbf{1}$ (on next page)}

Summary information on the training and test data used for model development and evaluation

*Median and range (minimum and maximum) are shown. SSD, species sensitivity

distribution; Class 3 or 4, reactive chemicals or specifically acting chemicals, respectively, based on the Verhaar mode of action classification (Verhaar, van Leeuwen \& Hermens 1992);

$\mathrm{SD}$, standard deviation; $\log K_{\text {ow }}, \log _{10}$-transformed octanol-water partition coefficient; log VP, $\log _{10}$-transformed vapor pressure at $25^{\circ} \mathrm{C}$ (see Materials and Methods section for further details). 
1 Table 1. Summary information on the training and test data used for model development and

2 evaluation

\begin{tabular}{lll}
\hline Variable & Training data & Test data \\
\hline Number of chemicals & 45 & 15 \\
Number of Class 3 chemicals & 15 & 7 \\
Number of Class 4 chemicals & 6 & 1 \\
Number of species* & $10(8-19)$ & $9(8-21)$ \\
SSD mean* & $0.92(-2.71-4.50)$ & $1.06(-1.05-4.40)$ \\
SSD SD* & $0.67(0.25-1.65)$ & $0.42(0.26-1.39)$ \\
Molecular weight* & $137(56-391)$ & $144(61-221)$ \\
Log $K_{\text {Ow }} *$ & $2.65(-1.47-7.43)$ & $2.18(-1.61-3.93)$ \\
Log VP* & $-1.54(-6.56-2.42)$ & $-0.91(-4.88-1.31)$ \\
Biodegradability* & $3.56(2.85-4.74)$ & $3.58(3.09-3.92)$ \\
\hline
\end{tabular}

3 *Median and range (minimum and maximum) are shown. SSD, species sensitivity distribution;

4 Class 3 or 4, reactive chemicals or specifically acting chemicals, respectively, based on the

5 Verhaar mode-of-action classification (Verhaar, van Leeuwen \& Hermens, 1992); SD, standard

6 deviation; $\log K_{\mathrm{OW}}, \log _{10^{-}}$-transformed octanol-water partition coefficient; $\log \mathrm{VP}, \log _{10^{-}}$

7 transformed vapor pressure at $25^{\circ} \mathrm{C}$ (see Materials and Methods section for further details). 


\section{Table 2 (on next page)}

Full-model-averaged standardized parameter estimates for the four models selected for predicting the mean and standard deviation (SD) of species sensitivity distribution (SSD)

Higher values of full-model-averaged standardized parameter estimates indicate higher relative importance of the variable in the model that was developed. Underlined predictors are those included in the best models. NA, not applicable. See the footnote to Table 1 for further details about the predictors. 
1 Table 2. Full-model-averaged standardized parameter estimates for the four models selected for

2 predicting the mean and standard deviation (SD) of species sensitivity distribution (SSD)

\begin{tabular}{|c|c|c|c|c|}
\hline \multirow{4}{*}{ Predictor } & \multicolumn{2}{|c|}{ Model for SSD mean } & \multicolumn{2}{|c|}{ Model for SSD SD } \\
\hline & & 3-species mean & & 3-species SD \\
\hline & Descriptors & & Descriptors & \\
\hline & & + descriptors & & + descriptors \\
\hline 3-species mean & NA & $\underline{0.938}$ & NA & NA \\
\hline 3-species SD & NA & NA & NA & $\underline{0.706}$ \\
\hline Biodegradability & 0.009 & 0.006 & -0.057 & -0.012 \\
\hline Class 3 & -0.191 & 0.002 & 0.004 & 0.005 \\
\hline Class 4 & $\underline{-0.580}$ & -0.025 & $\underline{0.687}$ & $\underline{0.231}$ \\
\hline $\log K_{\mathrm{OW}}$ & -0.349 & -0.003 & 0.020 & 0.005 \\
\hline Log VP & 0.049 & 0.019 & 0.138 & $\underline{0.107}$ \\
\hline Molecular weight & 0.016 & -0.018 & 0.093 & 0.012 \\
\hline
\end{tabular}

3 Higher values of full-model-averaged standardized parameter estimates indicate higher relative

4 importance of the variable in the model that was developed. Underlined predictors are those

5 included in the best models. NA, not applicable. See the footnote to Table 1 for further details 6 about the predictors. 


\section{Table 3 (on next page)}

Model evaluation for estimating the mean and standard deviation (SD) of species sensitivity distribution (SSD)

AICC, corrected Akaike information criterion; $r^{2}$, coefficient of determination; RMSE, root mean square error; $q^{2}$, leave-one-out cross-validated coefficient of determination; $\mathrm{RMSE}_{\mathrm{ext}}$, root

mean square error in the test data; $r_{\text {ext }}^{2}$ squared correlation coefficient between measured and predicted values; $Q^{2}{ }_{\text {ext, }}$ variance explained in the test data. 
1 Table 3. Model evaluation for estimating the mean and standard deviation (SD) of species

2 sensitivity distribution (SSD)

Models for SSD mean

Models for SSD SD

Statistics

3-species mean

3 species SD

Descriptors 3-species mean

Descriptors 3-species SD

+ descriptors $\quad+$ descriptors

$\begin{array}{lllllll}\text { AICc } & 121.7 & 20.0 & 19.5 & 5.0 & -19.3 & -22.4\end{array}$

$\underline{\text { Internal validation }}$

$\begin{array}{lllllll}r^{2} & 0.62 & 0.96 & 0.96 & 0.49 & 0.70 & 0.75\end{array}$

$\begin{array}{lllllll}\text { RMSE } & 0.82 & 0.28 & 0.27 & 0.24 & 0.18 & 0.17\end{array}$

$\begin{array}{lllllll}q^{2} & 0.52 & 0.95 & 0.95 & 0.41 & 0.67 & 0.69\end{array}$

$\underline{\text { External validation }}$

$\begin{array}{lllllll}r^{2}{ }_{\text {ext }} & 0.78 & 0.84 & 0.86 & 0.34 & 0.79 & 0.75\end{array}$

$\begin{array}{lllllll}\text { RMSE }_{\text {ext }} & 0.58 & 0.50 & 0.47 & 0.28 & 0.16 & 0.17\end{array}$

$\begin{array}{lllllll}Q^{2}{ }_{\text {ext }} & 0.75 & 0.84 & 0.86 & 0.25 & 0.69 & 0.66\end{array}$

\begin{tabular}{llllllll}
\hline \# of parameters & 3 & 1 & 2 & 1 & 1 & 3
\end{tabular}

3 AICc, corrected Akaike information criterion; $r^{2}$, coefficient of determination; RMSE, root mean

4 square error; $q^{2}$, leave-one-out cross-validated coefficient of determination; $\mathrm{RMSE}_{\text {ext }}$, root mean

5 square error in the test data; $r_{\text {ext }}^{2}$, squared correlation coefficient between measured and predicted

6 values; $Q^{2}$ ext, variance explained in the test data. 